\title{
Semillas de Spondias tuberosa oriundas de frutos cosechados en cuatro estadios de maduración y almacenadas ${ }^{1}$
}

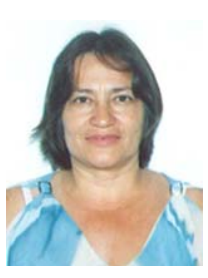

\author{
Adalgisa A. de Souza², Riselane de L. A. Bruno ${ }^{3}$, Kilson P. Lopes², Gleibson D. Cardoso², Walter E. \\ Pereira ${ }^{3} \&$ Jorge Cazé Filho ${ }^{4}$ \\ 1 Trabajo de la primera actora presentado al Curso de Pos Graduación en Agronomía del CCA/UFPB, Campus II, Areia, PB \\ ${ }^{2}$ CCA/UFPB. Campus II, Areia, PB. CEP 58 397-000. Fone: (83) 9382-7345. E-mail: adaaranha@yahoo.com.br (Foto), \\ kilsonlopes@terra.com.br, gleibson@cnpa.embrapa.br. \\ ${ }^{3}$ CCA/UFPB. Fone: (83) 3362-2300. E-mail: lane@cca.ufpb.br, wepereira@cca.ufpb.br \\ ${ }^{4}$ EMEPA, PB. Eurípedes Tavares, 210 Tambiá, Centro CEP 58.013-290. João Pessoa, PB. E-mail: cazéfilho@emepa.br
}

Protocolo 33 - 19/3/2004 - Aprovado em 24/2/2005

\begin{abstract}
Resumen: Las condiciones climatológicas que son peculiares de la región semiárida ofrece de forma natural plantas propicias a la explotación humana, las cuales se destaca la Spondias tuberosa Arruda Câmara, cuyo fruto es cosechado de forma extractivita y desordenada, poniendo en riesgo su perpetuación. El propósito de esta investigación fue analizar el tiempo de almacenamiento de semillas, el ambiente y el estadio de maduración del fruto que sea más adecuado a una buena propagación de esa especie fructífera. El ensayo se llevó a cabo en casa de vegetación del Centro de Ciencias Agrarias, CCA, Areia, PB. Se utilizó un diseño experimental de bloques al azar, en esquema factorial $5 \times 2 \times 4$, con cuatro repeticiones. Las variables estudiadas fueron el porcentaje de germinación, vigor, número de brotes, altura de plantas, diámetro y longitud de túbera. Semillas mantenidas bajo condiciones ambientes, durante ocho meses de almacenamiento alcanzaron mayor uniformidad de germinación, potencial germinativo $(90 \%)$ y vigor $(0,505)$. Las semillas más adecuadas a la optimización de buena germinación fueron aquéllas oriundas de frutos maduros $(87 \%)$ y muy maduros $(90 \%)$.
\end{abstract}

Palabras clave: umbucero, ambiente, vigor, germinación

\section{Seeds of Spondias tuberosa originated from fruits harvested at four maturation stages and stored}

\begin{abstract}
The peculiar climatic conditions of the semi-arid area offer plants for the human exploration in natural a way among these, stands out the Spondias tuberosa Arruda Câmara, whose fruit is havested in an extractive and disorganized way, putting in risk its perpetuation. The objective of this study was to verify the storage period of the seeds, the environments and the stage of fruit maturation most adopted to a good propagation of the species. The experiment was conduted in a greenhouse at the Center of Agricultural Sciences, (CCA), Areia, UFPB, PB. The experimental design used was a completely randomized in a factorial scheme $(5 \times 2 \times 4)$, with four repetitions. The studied variables were the germination percentage, vigour (index of germination speed), number of sprouts, height of plants, diameter and length of the tubers. The seeds maintained under natural environmental conditions, stored for a period of eight months presented higher germination uniformity, germinative potential of $90 \%$, and vigour of 0,505 . The most appropriate seeds to optimise germination were those originating from the mature fruits $(87,5 \%)$ and highly ripened fruits $(90 \%)$.
\end{abstract}

Key words: umbuzeiro, environments, vigour, germination

\section{INTRODUCCIÓN}

La peculiaridad de las condiciones climáticas de la región semiárida ofrece de forma natural plantas propicias a la explotación humana, destacándose entre éstas, la Spondias tuberosa Arruda Câmara, cuyo fruto es explotado de forma extractivita y desordenada, poniendo en riesgo su perpetuación por la cosecha excesiva de sus frutos en su hábitat, debido a su principal vía de propagación ser la semilla.

La adaptación de ésta a distintas condiciones, la considerable producción por planta, la extraordinaria resistencia a los periodos de seca y la alta aceptabilidad de sus frutos en la 
zona de dispersión natural y en los grandes centros, constituyen una imperiosa necesidad de pesquisas relativas a esta especie fructífera.

El extractivismo de la Spondias sp como fuente alternativa de rentabilidad para los pequeños productores del semiárido, alcanza $80 \%$ de las familias de la comunidad, donde hay grupos distintos de personas cuanto al periodo y al tiempo dedicados a esa actividad y, principalmente, cuanto a la renta alcanzada con la venta de sus frutos (Cavalcanti et al., 1996). De acuerdo con Silva et al. (1998), este árbol es de grande expresión económica para la micro región del Agreste. Sus raíces son superficiales, pero, muy desarrolladas, quedando su totalidad en los primeros $100 \mathrm{~cm}$ del suelo. Presentan órganos de reservas llamados xilopódios (túberas), que son estructuras entumecidas, de consistencia esponjosa, redonda, oscura, alcanzando cerca de $4 \mathrm{~kg}$ de peso, constituída por tejido lagunoso, celulósico y lleno de agua (Mendes, 1990), siendo su superviviencia en condiciones adversas, mantenida no sólo por esos órganos, sino también, por la pérdida de las hojas en el verano y metabolismo fisiológico del cierre de los estomas en las horas más calientes del día, a lo que se les atribuye regulación del agua, ejerciendo rígido control en la transpiración, garantizando así, su extraordinaria resistencia a los periodos secos que les son comunes anualmente.

En pesquisas más recientes para evaluar la productividad de 36 plantas nativas de Spondias tuberosa en distintas comunidades de la región semiárida del Estado de Bahia, Cavalcanti et al. (1999) obtuvieron un promedio de producción yendo de 18.853 a 21.366 frutos por planta; con estos datos, los mismos garantizan que esta especie puede contribuir satisfactoriamente para el desarrollo de la región nordeste brasileña, por el aprovechamiento racional de su fruto, principalmente porque se ha registrado disminución de la densidad de esa cultura en el semiárido. Las especies nativas de Brasil, son exploradas localmente y, muchas veces de forma extractivas; por eso, debido a los factores de desarrollo, como la expansión de las fronteras agrícolas y al uso de cultivares mejoradas para atender a las necesidades de producción de alimentos, viene ocurriendo una pérdida de gran valía de germoplasmas, lo que llevará en algunos casos a la extinción de especies.

En Spondias sp, la propagación se hace generalmente de forma sexuada, dónde el endocarpio es la unidad de dispersión, que por su constitución impermeabilizante, ejerce cierta dormencia llevando a una germinación lenta y desuniforme, dificultando la emergencia de los plantines, conforme fue verificado por algunos actores (Silva \& Silva, 1974; Mendes, 1990; Gondim et al., 1991; Campos, 1996; Souza, 1999; Costa, 1999; Souza, et al. 2000). La durmencia de los endocarpios ocurre principalmente por la resistencia mecánica de éste a la expansión del embrión y un corte en forma de bisel en la parte distal del endocarpio es eficiente en la superación de ésa durmencia, contribuyendo para la uniformización de la germinación (Campos, 1996).

Evaluándose la madurez adecuada para semillas oriundas de frutos de Spondias tuberosa, Costa (1999) constató que aquéllas procedentes de frutos maduros y en maduración adelantada originaron plantas con mayor altura y peso de materia seca de la parte aérea, habiendo formación de xilopódio en todas las plantas estudiadas. De acuerdo con Araujo et al. (2001), semillas con mayor periodo de almacenamiento presentaron los mejores porcentajes de germinación $(73,6 \%)$, pero, el diámetro y longitud de las raíces, peso total de plántulas y número de brotes, no fueron afectados con el pasar del tiempo.

Con el fin de atender a las necesidades de la creciente población mundial, sin destruir, cada vez más, la débil ecología de nuestro planeta, hay que procurar medios y métodos más eficientes que aumente la producción de alimentos. En este contexto, se busca a través de este trabajo, determinar el periodo de almacenamiento de semillas, el ambiente y el estadio de maduración de frutos más adecuado para viabilizar la propagación de Spondias tuberosa.

\section{MATERIAL Y MÉTODOS}

El ensayo se llevó a cabo con semillas (endocarpios) de Spondias tuberosa en condiciones de casa de vegetación del Centro de Ciencias Agrarias de la Universidad Federal de Paraíba, Areia, PB.

Los frutos fueron cosechados de plantas en el distrito de Soledade, PB, micro región del Seridó Paraibano, en cuatro estadios de maduración: fruto verde $\left(E_{1}\right)$, en inicio de maduración $\left(\mathrm{E}_{2}\right)$, maduro $\left(\mathrm{E}_{3}\right)$ y muy maduro, cuando echados al suelo $\left(\mathrm{E}_{4}\right)$.

En la obtención de las semillas, se utilizó una máquina despulpadora para la extracción de la cáscara y pulpa de los frutos. Posteriormente, las mismas fueron puestas sobre un papel periódico, en ambiente al aire libre protegidos de luz solar y después, acondicionadas en embalaje de papel empaque. Los tratamientos correspondieron a cada uno de los estadios de maduración; una parte fue almacenada en condiciones bajo control (cámara) a temperatura de $20 \pm 3{ }^{\circ} \mathrm{C}$, y humedad relativa del aire de 70 a $79 \%$ y la otra parte, en condiciones ambientes (laboratorio) a una temperatura de 26 a $30{ }^{\circ} \mathrm{C}$ y humedad relativa del aire de 59 a $70 \%$, a lo largo de ocho meses (Figura 1).

Después de la instalación del ensayo (11/3/2002) y a cada dos meses de almacenadas, las semillas fueron sembradas en la posición vertical, en bandejas plásticas midiendo 39,5 x 27,5 x $10 \mathrm{~cm}$, en sustrato de arena lavada, con profundidad media de $2,5 \mathrm{~cm}$, con cuatro repeticiones de diez semillas por unidad experimental (parcelas) y se regó periódicamente, manteniéndose el sustrato siempre húmedo.

Las variables estudiadas fueron el porcentaje de germinación (GER), el índice de vigor (IVE, obtenido por la sumatoria de los cocientes resultantes de dividir el número de plantines normales entre los días de puesta a germinar la semilla), número de brotes (NB), altura de planta (AP), diámetro (DX) y largura de túbera (CX). Los datos de germinación y índice de vigor fueron registrados a partir del inicio de la germinación de las semillas provenidas de cada periodo de almacenamiento $(0,2,4,6$ y 8 meses $)$, siguiéndose anotaciones periódicas hasta estabilidad germinativa (150 días), tras la siembra. Las medidas de longitud y diámetro de túberas fueron determinadas con una regla y parquímetro graduado, respectivamente. 

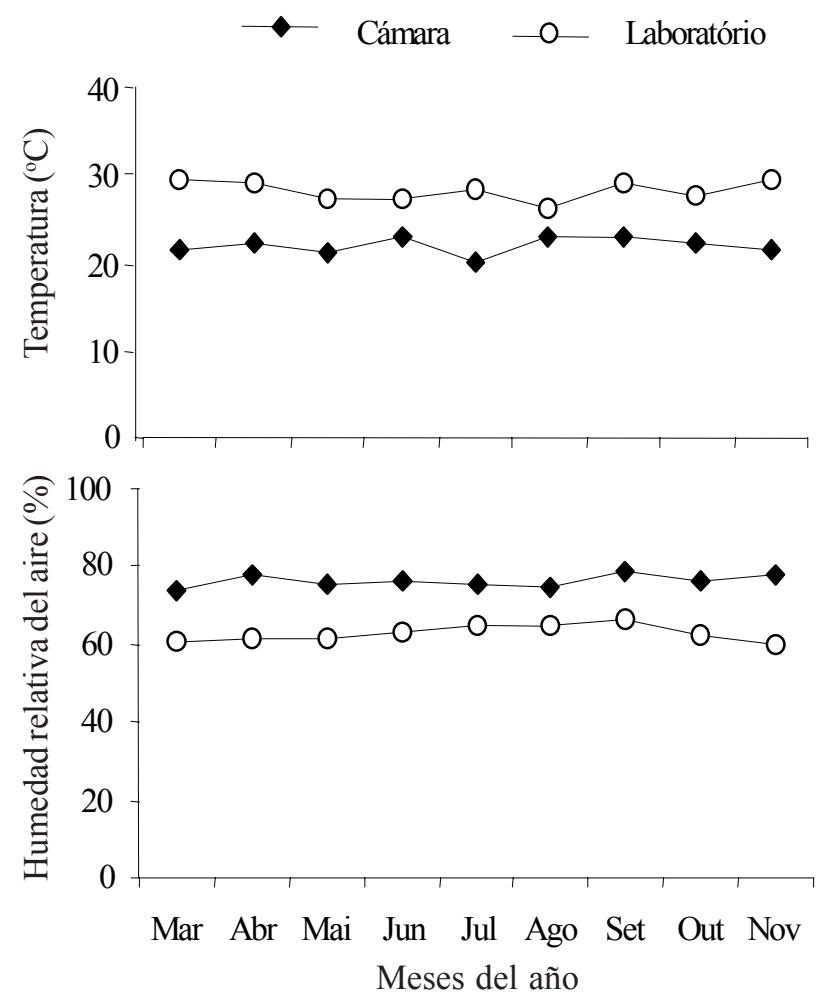

Figura 1. Promedios de temperatura y humedad relativa del aire, registrados en los ambientes que contenían las semillas almacenadas de Spondias tuberosa, oriundas de frutos cosechados en cuatro estadios de maduración

Se hizo uso de un diseño experimental de bloques al azar, en esquema factorial $(5 \times 2 \times 4)$, cinco períodos de almacenamiento $\left(T_{1}, T_{2}, T_{3}, T_{4}, T_{5}\right)$, dos ambientes $\left(A_{1}, A_{2}\right)$ y cuatro estadios de maduración $\left(\mathrm{E}_{1}, \mathrm{E}_{2}, \mathrm{E}_{3}, \mathrm{E}_{4}\right)$, con cuatro repeticiones. Finalmente se ajustaron modelos de regresión a las variables mencionadas en función del tiempo de almacenamiento de la semilla, con valores promedios porcentuales transformados en arc sen $\sqrt{x / 100}$ y comparados por Tukey a $5 \%$ de probabilidad.

\section{RESULTADOS Y DISCUSIÓN}

En la Tabla 1, se observa los resultados alcanzados en este experimento los cuáles indican respuesta significativa para los factores aislados (tiempo de almacenamiento, maduración y ambiente), así como, para las interacciones.

De acuerdo con los datos obtenidos, la germinación de las plántulas de Spondias sp fue muy baja y irregular en principio, con emergencia de la primera plántula a los 27 días tras la siembra, reduciéndose linealmente a partir de entonces, hasta 10 días tras la siembra en el final del experimento (ocho meses).

Los valores de germinación de semillas de frutos cosechados en inicio de maduración, maduros y muy maduros, alcanzaron mayor porcentaje, principalmente cuando almacenadas bajo condiciones ambientes, con valores no significativos para semillas oriundas de frutos verdes y muy maduros, entre ambientes (Figura 2A).

Resultados semejantes fueron registrados por Costa (1999) que observó baja germinación para semillas oriundas de frutos
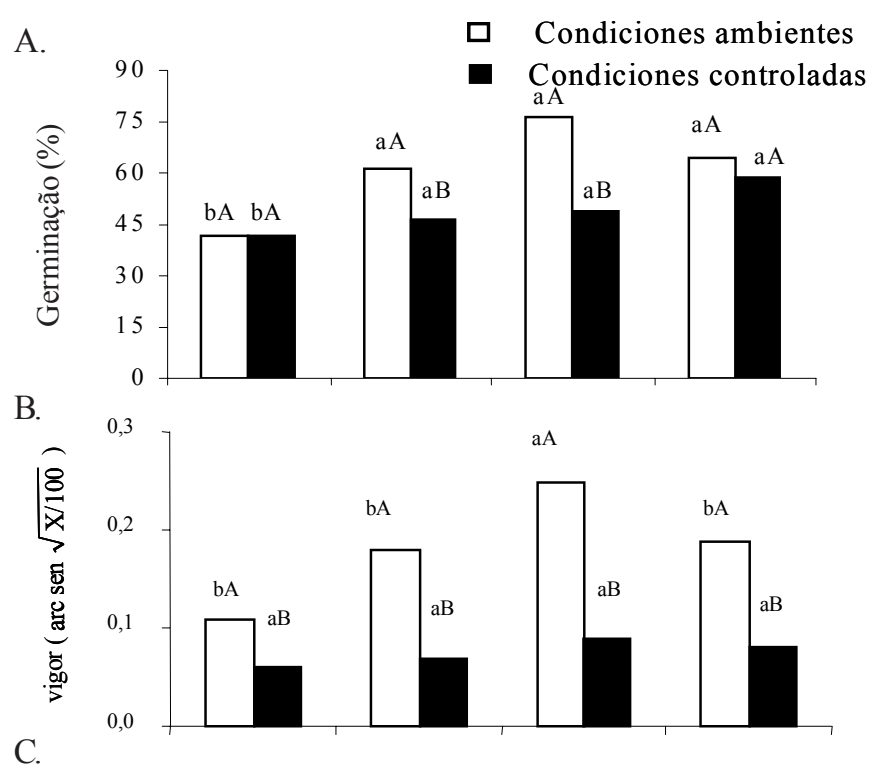

C.

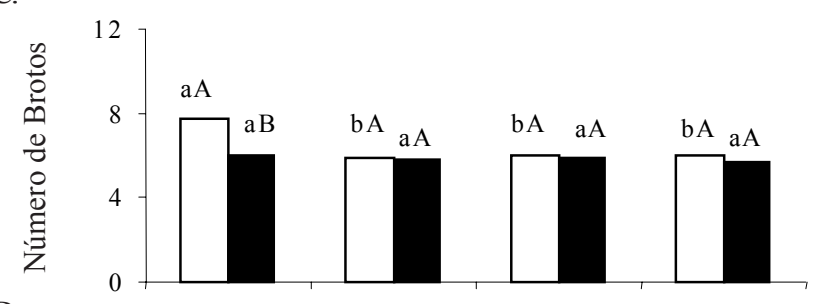

D.

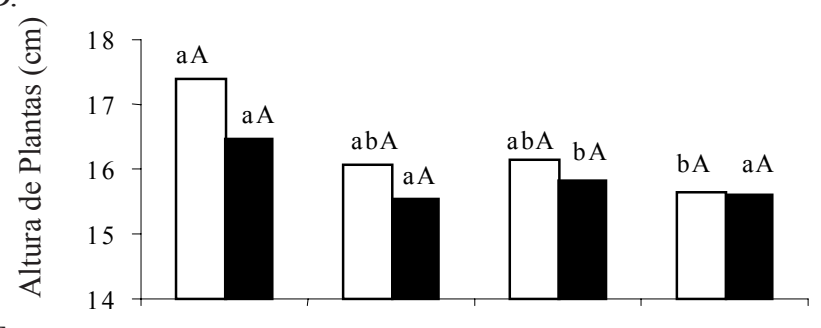

E.

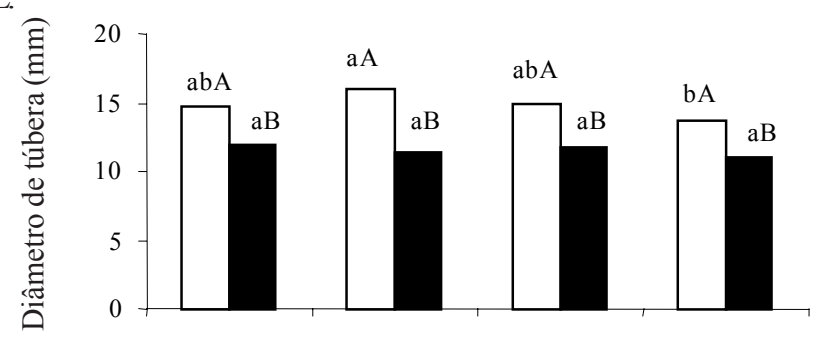

F.

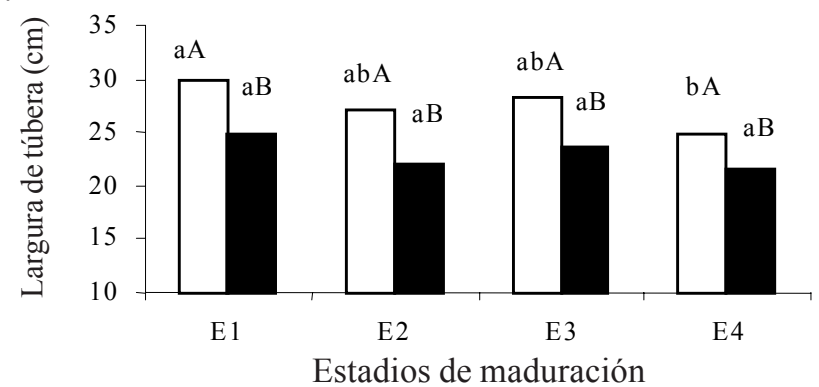

$\mathrm{E}_{1}$ - Fruto verde; $\mathrm{E}_{2}$ - Fruto en inicio de maduración; $\mathrm{E}_{3}$ - Fruto maduro; $\mathrm{E}_{4}$ - Fruto muy maduro Obs.: Promedios seguidos por una misma letra minúscula (entre ambientes) y mayúscula (entre estadios de maduración), no difieren entre sí por el teste de Tukey a $5 \%$ de probabilidad

Figura 2. Calidad fisiológica y agronómica para la interacción estadio de maduración x ambiente de semillas de Spondias $\mathrm{sp}$, oriundas de frutos cosechados en cuatro estadios de maduración y almacenadas 
Tabla 1. Análisis y coficiente de variación (CV) para emergencia (GER), índice de vigor (IVE), número de brotes (NB), altura de planta (AP), diámetro (DX) y longitud de túbera (CX) de semillas de Spondias tuberosa, oriundas de frutos cosechados en cuatro estadios de maduración y almacenadas

\begin{tabular}{|c|c|c|c|c|c|c|c|}
\hline \multirow{2}{*}{$\begin{array}{l}\text { Fuentes de } \\
\text { Variación }\end{array}$} & \multirow[b]{2}{*}{ GL } & \multicolumn{6}{|c|}{ Quadrado Médio } \\
\hline & & GER (\%) & IVE & NB & $\mathrm{AP}(\mathrm{cm})$ & $\mathrm{DX}(\mathrm{mm})$ & $\mathrm{CX}(\mathrm{cm})$ \\
\hline Bloques & 3 & 0,014 & 0,0006 & 1,663 & 1,870 & 1,120 & 17,080 \\
\hline Almacen. $-\mathrm{T}$ & 4 & $1,30 * *$ & $0,20 * *$ & $141,56^{* *}$ & $307,41^{* *}$ & $326,18^{* *}$ & $477,68 * *$ \\
\hline Maturación - E & 3 & $0,44 * *$ & $0,05^{* *}$ & $9,51 * *$ & $13,85^{* *}$ & $13,16^{\mathrm{ns}}$ & $127,27 * *$ \\
\hline Ambiente - A & 1 & $0,77 * *$ & $0,45 * *$ & $15,36^{* *}$ & $8,24^{\mathrm{ns}}$ & $422,73^{* *}$ & $834,02 * *$ \\
\hline $\mathrm{T} \times \mathrm{E}$ & 12 & $0,09 * *$ & $0,01 * *$ & $1,32^{\mathrm{ns}}$ & $4,99^{\mathrm{ns}}$ & $20,76^{* *}$ & $21,49^{\mathrm{ns}}$ \\
\hline $\mathrm{T} \times \mathrm{A}$ & 4 & $0,20 * *$ & $0,06 * *$ & $2,30^{\mathrm{ns}}$ & $8,09^{*}$ & $11,46^{\mathrm{ns}}$ & $125,65 * *$ \\
\hline $\mathrm{E} \times \mathrm{A}$ & 3 & $0,13 * *$ & $0,02^{* *}$ & $5,90 * *$ & $1,52^{\mathrm{ns}}$ & $8,54^{\text {ns }}$ & $6,63^{\mathrm{ns}}$ \\
\hline $\mathrm{T} \times \mathrm{E} \times \mathrm{A}$ & 12 & $0,03^{\mathrm{ns}}$ & $0,0016^{\mathrm{ns}}$ & $2,33 * *$ & $10,49^{* *}$ & $16,54 * *$ & $72,46^{* *}$ \\
\hline Tiempo d. $A_{1}$ & (4) & & & & & & \\
\hline Linear & 1 & $4,25^{* *}$ & $0,927 * *$ & $287,75^{* *}$ & $787,43^{* *}$ & $538,39 * *$ & $461,72 * *$ \\
\hline Cuadrática & 1 & $0,61 * *$ & $0,0002^{\mathrm{ns}}$ & $42,04 * *$ & $1,863^{\mathrm{ns}}$ & $31,365^{*}$ & $0,885^{\mathrm{ns}}$ \\
\hline Cúbica & 1 & $0,06^{\mathrm{ns}}$ & $0,017 * *$ & $1,36^{\mathrm{ns}}$ & $29,24 * *$ & $140,25^{* *}$ & $1.166,13^{* *}$ \\
\hline Tiempo d. $A_{2}$ & (4) & & & & & & \\
\hline Linear & 1 & $0,81^{* *}$ & $0,079^{* *}$ & $172,6^{* *}$ & $414,64^{* *}$ & $271,94 * *$ & $0,344^{\mathrm{ns}}$ \\
\hline Cuadrática & 1 & $0,19 * *$ & $0,0006^{\mathrm{ns}}$ & $56,82 * *$ & $0,563^{\mathrm{ns}}$ & $43,746 * *$ & $0,0052^{\mathrm{ns}}$ \\
\hline Cúbica & 1 & $0,03^{\mathrm{ns}}$ & $0,0049^{\mathrm{ns}}$ & $1,84^{\mathrm{ns}}$ & $14,07^{\mathrm{ns}}$ & $39,036 * *$ & $109,87^{*}$ \\
\hline Falta de ajuste & (2) & $0,03^{\mathrm{ns}}$ & $0,013^{\mathrm{ns}}$ & $6,525^{*}$ & $7,095^{\text {ns }}$ & $142,92 *$ & $337,18^{*}$ \\
\hline Tiempo d. $E_{1}$ & (4) & & & & & & \\
\hline Linear & 1 & $0,123^{*}$ & $0,062 * *$ & $125,37 * *$ & $369,46^{* *}$ & $201,55^{* *}$ & $184,07 * *$ \\
\hline Cuadrática & 1 & $0,019^{\mathrm{ns}}$ & $0,0011^{\mathrm{ns}}$ & $31,196^{* *}$ & $0,857^{\mathrm{ns}}$ & $0,160^{\mathrm{ns}}$ & $0,072^{\mathrm{ns}}$ \\
\hline Cúbica & 1 & $0,166^{* *}$ & $0,014 * *$ & $0,220^{\mathrm{ns}}$ & $0,336^{\mathrm{ns}}$ & $0,032^{\mathrm{ns}}$ & $155,68^{*}$ \\
\hline Tiempo d. $E_{2}$ & (4) & & & & & & \\
\hline Linear & 1 & $1,905^{* *}$ & $0,224 * *$ & $127,39 * *$ & $212,16^{* *}$ & $110,38^{* *}$ & $24,12^{\mathrm{ns}}$ \\
\hline Cuadrática & 1 & $0,497^{* *}$ & $0,0004^{\mathrm{ns}}$ & $25,108 * *$ & $1,010^{\mathrm{ns}}$ & $28,856^{*}$ & $4,039^{\mathrm{ns}}$ \\
\hline Cúbica & 1 & $0,049^{\mathrm{ns}}$ & $0,0030^{\mathrm{ns}}$ & $0,882^{\mathrm{ns}}$ & $16,254 *$ & $98,32 * *$ & $344,90 * *$ \\
\hline Tiempo d.E $E_{3}$ & (4) & & & & & & \\
\hline Linear & 1 & $1,238^{* *}$ & $0,260 * *$ & $117,98^{* *}$ & $374,80^{* *}$ & $418,38 * *$ & $105,66^{*}$ \\
\hline Cuadrática & 1 & $0,397 * *$ & $0,0013^{\mathrm{ns}}$ & $20,614 * *$ & $0,019^{\mathrm{ns}}$ & $18,737^{\mathrm{ns}}$ & $5,220^{\mathrm{ns}}$ \\
\hline Cúbica & 1 & $0,0014^{\mathrm{ns}}$ & $0,0042^{\mathrm{ns}}$ & $0,428^{\mathrm{ns}}$ & $63,938 * *$ & $106,49 * *$ & $478,88^{* *}$ \\
\hline Tiempo d. $\mathrm{E}_{4}$ & (4) & & & & & & \\
\hline Linear & 1 & $1,810^{\mathrm{ns}}$ & $0,276^{* *}$ & $85,07 * *$ & $235,16^{* *}$ & $120,51 * *$ & $6,050^{\mathrm{ns}}$ \\
\hline Cuadrática & 1 & $0,060^{\mathrm{ns}}$ & $0,00007^{\mathrm{ns}}$ & $22,04 * *$ & $0,847^{\mathrm{ns}}$ & $63,586^{* *}$ & $33,53^{\text {ns }}$ \\
\hline Cúbica & 1 & $0,0062^{\mathrm{ns}}$ & $0,0022^{\mathrm{ns}}$ & $2,261^{\mathrm{ns}}$ & $0,117^{\mathrm{ns}}$ & $30,541 *$ & $103,74^{*}$ \\
\hline Falta de Ajuste & (4) & $0,04^{\mathrm{ns}}$ & $0,015^{*}$ & $12,88^{*}$ & $7,27 *$ & $193,41^{*}$ & $361,32 *$ \\
\hline Resíduo & 117 & 0,021 & 0,0018 & 0,967 & 2,961 & 5.601 & 24.289 \\
\hline Promedio real & & 84,0 & 0,13 & 6,10 & 16,08 & 13,15 & 25,25 \\
\hline CV (\%) & & 17,37 & 32,46 & 16,11 & 10,70 & 19,52 & 18,00 \\
\hline
\end{tabular}

* y ** significativo a 5 y $1 \%$ de probabilidad, respectivamente, por el teste $\mathrm{F}$; ns no significativo

verdes, lo que justificó ser debido a una probable inmadurez fisiológica de la semilla, lo que está de acuerdo con Alvarenga et al. (1984), cuando los mismos garantizan que semillas oriundas de frutos verdes pueden alcanzar capacidad germinativa, pero, vigor reducido.

Para el índice de vigor determinado por la velocidad de germinación, el más alto valor fue obtenido por semillas oriundas de frutos maduros, evidenciándose que, en todos los estadios de maduración, aquéllas que fueron almacenadas bajo condiciones ambientes fueron las más vigorosas (Figura 2B). Cuanto a los brotes, el mayor número por planta fue verificado en aquéllas remanecientes de semillas provenientes de frutos en el estadio de maduración verde, diferenciándose de los demás estadios, cuando mantenidas bajo condiciones normales de temperatura y humedad relativa; pero en condiciones controladas (cámara), esos valores no fueron significativos entre si, ni entre semillas oriundas de frutos en inicio de maduración, maduros y muy maduros (Figura 2C). Comportamiento semejante fue verificado para la variable altura de planta en este mismo estadio de maduración, no habiendo diferencias significativas en los estadios en inicio de maduración y maduros, ni entre ambientes (Figura 2D). En un otro experimento, evaluándose la madurez probable para semillas de Spondias tuberosa, Costa (1999) verificó que aquéllas provenientes de frutos maduros y en estadio de maduración avanzado originaron plantas con mayor altura y peso de materia seca de la parte aérea. Ya con relación al diámetro, el mayor fue obtenido por plantas provenientes de 
semillas oriundas de frutos en inicio de maduración seguido de aquellos maduros y verdes, con diferencia significativa para semillas oriundas de frutos muy maduros, principalmente cuando éstas fueron almacenadas bajo condiciones ambientes ( los estadios de maduración (Figura 2E).

Igual comportamiento fue registrado para longitud de túbera, con efecto no significativo entre ambientes, en ninguno de los estadios de maduración evaluados (Figura 2F). Observase en este trabajo que las semillas oriundas de frutos verdes presentaron valores significativamente mayores para las características número de brotes y altura de plantas, siendo semejantes a los otros estadios de maduración en la variable diámetro y longitud de la túbera (Figuras $2 \mathrm{C}$ e D). Lo que puede ser justificado porque las plantas evaluadas en este tiempo, haberen sido aquèllas remanecientes de la germinación, no habiendo, por lo tanto, relación lineal con la emergencia inicial, ni con el índice de vigor, cuyos valores para este estadio fueron significativamente más reducidos (Figura 2B).

Como se sabe, la curva de maduración de Spondias sp, a partir de la apertura de la flor hasta el completo amadurecimiento del fruto, corresponde a un promedio de tiempo de 124 días (Martins et al., 2003a) y como esos frutos fueron cosechados ya tardíamente, probablemente, ya estaban aptos a la germinación. De acuerdo con Cavalcanti et al. (2004), el periodo medio en esta especie fructífera a partir del inicio de fructificación hasta la maduración total del fruto, es de 125 días; todavía, plantas más precoces presentan frutos maduros a los 117 días después de la fructificación.

En frutos de cajá (Spondia mombin L.), el periodo de crecimiento más rápido ocurrió en el estadio verde de maduración, debido a intensa actividad meristemática (Lozano, 1986); así como, en frutos de Spondias purpurea, la fase de crecimiento mayor fue sumada a una pigmentación verdeintensa, con características indicativas de actividad celular máxima, reflejada por la inmadurez fisiológica; así que, frutos unidos a planta madre, en el estadio totalmente verde, llegan al inicio de su maduración a los 14 días, a la madurez y amadurecimiento, 4 días después (Martins et al., 2003b). En todas las variables evaluadas los mayores valores alcanzados fueron por aquéllas semillas que estuvieron almacenadas bajo condiciones ambientes.

\section{Efecto del almacenamiento en la calidad de semillas de Spondias tuberosa (T x E)}

Las semillas mantenidas por más tiempo almacenadas, alcanzaron porcentaje germinativo significativamente mayor, cuya variación pasó de 10 a $30 \%$ en el inicio del experimento hasta 90 a $100 \%$ (datos reales) a los ocho meses del almacenamiento, principalmente en aquéllas oriundas de frutos en los estadios iniciales de maduración, maduros y muy maduros (Figura 3A), dónde se puede inferir gran influencia del tiempo pasado entre la cosecha y la siembra de las mismas en la velocidad de emergencia de las semillas (Figura 3B). Conforme se verificó en ese trabajo, cuyo índice de vigor pasó de 0,024 en la fase inicial de instalación del trabajo, para 0,505 en el final del experimento (datos reales), principalmente bajo condiciones ambientes y en el estadio de maduración maduro.
A.

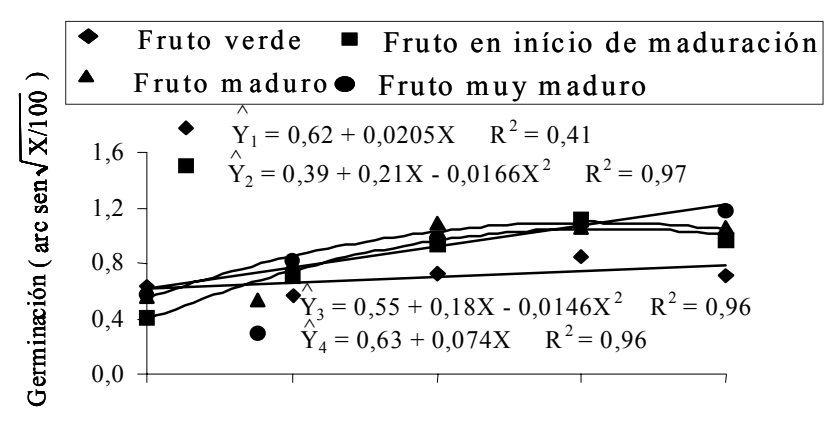

B.

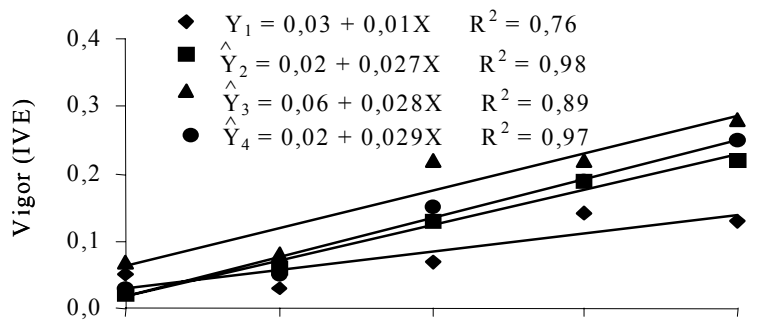

C.

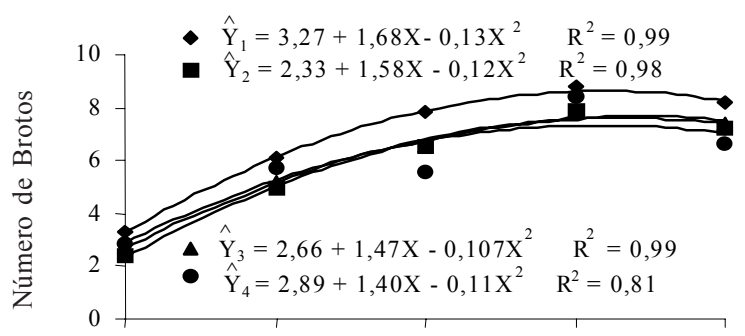

D.

E.

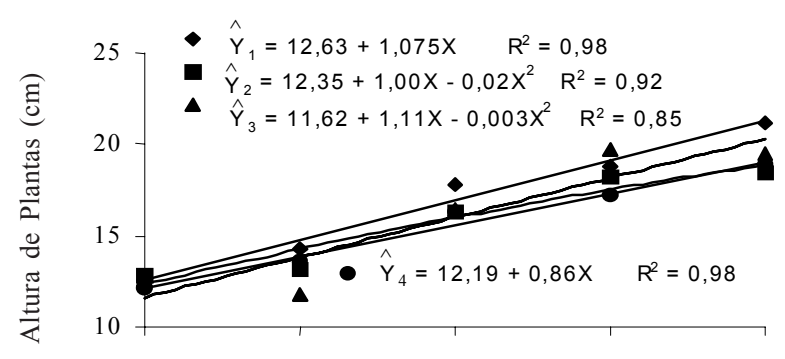

F.
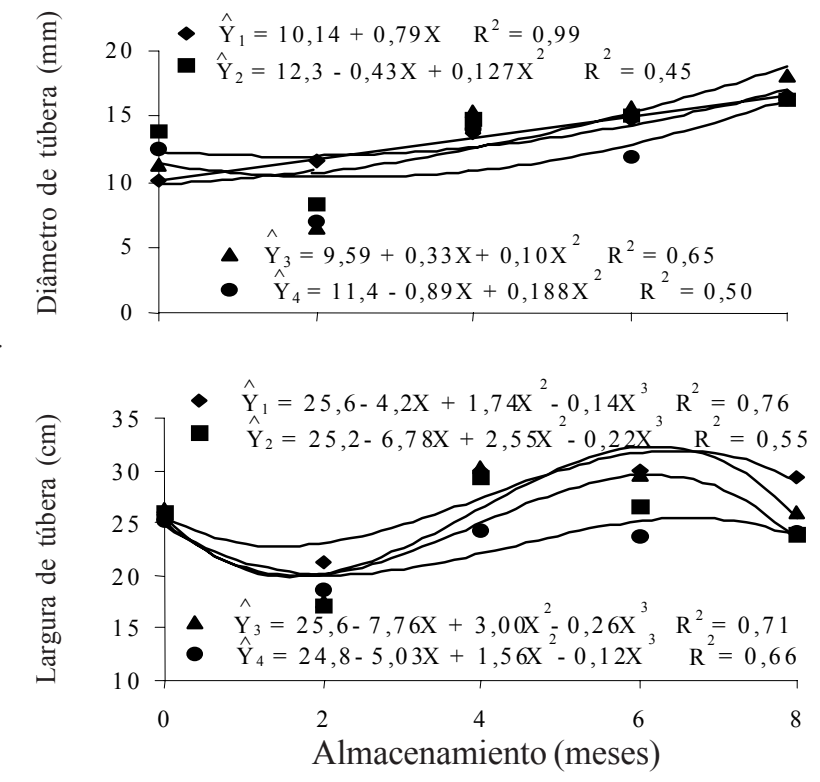

Figura 3. Calidad fisiológica y agronómica para la interacción tiempo de almacenamiento x estadio de maduración, de semillas de Spondias tuberosa oriundas de frutos cosechados en cuatro estadios de maduración y almacenadas 
Resultados semejantes fueron obtenidos por Lederman et al. (1989), para la emergencia de plántulas. Así como, Araujo et al. (2001) también evidenciaron porcentuales germinativos crecientes respectivos de $22,8,27,7$ y $73,6 \%$ para semillas de Spondias tuberosa a los 0, 12 y 24 meses del almacenamiento. Estos mismos autores constataron que la emergencia de semillas de Spondias sp. se hizo influenciable no sólo con la quiebra de durmencia, pero también, con el tiempo de almacenamiento.

Vale resaltar todavía que, en el presente estudio, la primera emergencia de las plántulas fue a los 27 días después de la siembra (datos no publicados), reduciéndose a partir de entonces, en cada uno de los períodos de almacenamiento, hasta 10 días en el período final de la investigación, o sea, ocho meses, constatándose de esa forma, una capacidad germinativa mayor y aún, mejor uniformidad al paso del tiempo, justificado por el índice de vigor alcanzado en el inicio y final del experimento (Figura 3B).

Con relación al número de brotes (Figura 3C), hubo superación del estadio de maduración verde, con el mismo comportamiento en todos los estadios de maduración a los seis meses de almacenadas. Por otro lado, para altura de plantas (Figura 3D), hubo un aumento lineal en todos los estadios de maduración con el paso del tiempo, evidenciándose eso, principalmente, en el estadio verde de maduración. Para diámetro de túbera (Figura 3E) hubo efecto cuadrático en todos los estadios de maduración evaluados, con mayores valores a partir del según mes hasta el último periodo de la evaluación del teste; en contrapartida, para la variable longitud de túbera (Figura 3F), inicialmente hubo semejanza con el diámetro, pero, alrededor de los seis meses, hubo una ligera disminución, debido posiblemente a factores físicos, tales como, impedimento del crecimiento longitudinal de la raíz, por ello ser un factor limitante al desarrollo normal de la túbera.

Efecto del ambiente en el almacenamiento de semillas de Spondias tuberosa (T x A)

Para las variables germinación y vigor (Figura 4A e B), hubo un mayor incremento de valores a partir de los cuatro meses del almacenamiento, siempre con promedio más alto en semillas mantenidas en condiciones ambientes, principalmente a partir de los seis meses del almacenamiento; lo que va de encuentro a lo descrito por Araujo et al. (2001), cuando estos constataron que semillas con mayor periodo de almacenamiento presentaron mayor porcentaje y uniformidad de germinación. En ese caso, se verificó semillas con un promedio de germinación de $90 \%$ (dados reales) a partir de los seis meses de almacenadas, en condiciones ambientes (Figura 4A).

Para el índice de vigor (Figura 4B), las semillas almacenadas por ocho meses, fueron las más vigorosas, principalmente en condiciones ambientes, lo que se lleva a creer en una posible adaptabilidad de esa cultura a las condiciones ambientales no controladas de temperatura más elevada y humedad relativa del aire variable, o sea, ambientes que tengan una mayor representatividad de su hábitat natural, como se verificó en las oscilaciones ocurridas durante todo el tiempo del almacenamiento (Figura 1).

Cuanto al número de brotes (Figura 4C), se observó efecto cuadrático, y mayores valores a partir de los seis meses de

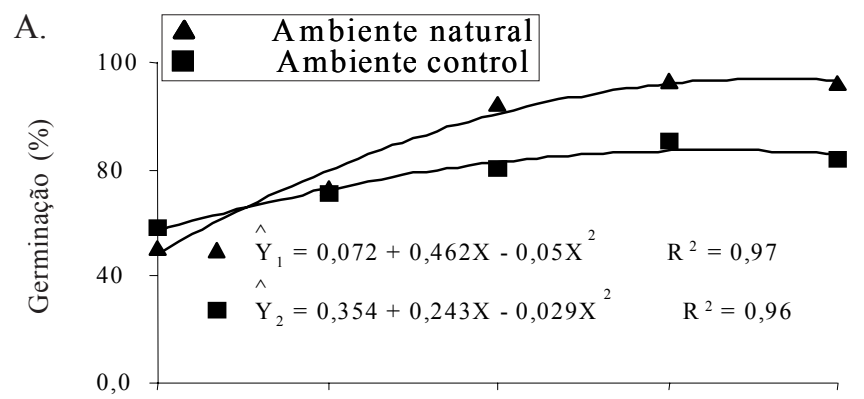

B.

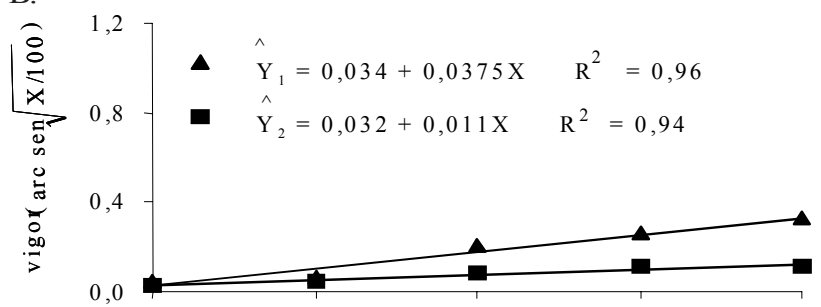

C.
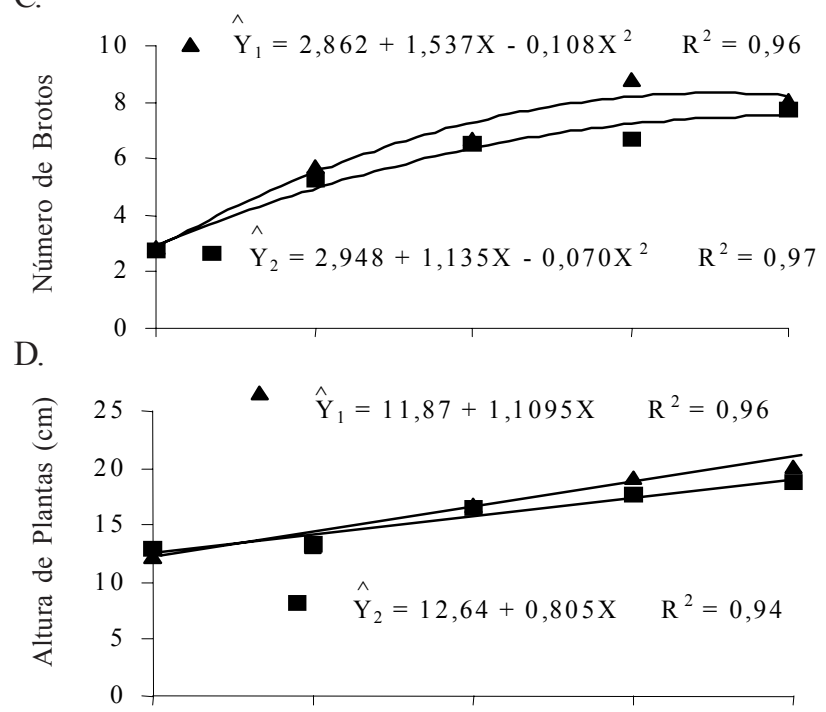

$\mathrm{E}$.

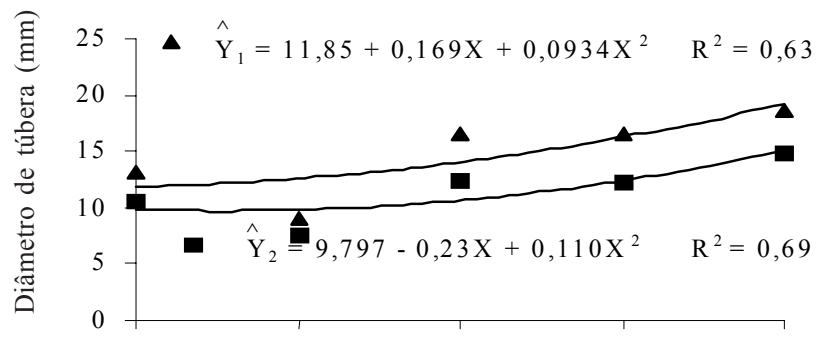

F.

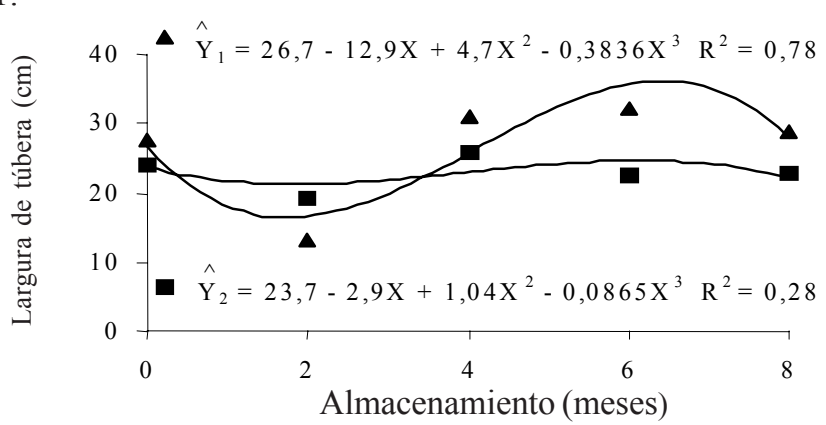

Figura 4. Calidad fisiológica y agronómica para la interacción tiempo de almacenamiento $\mathrm{x}$ ambientes, de semillas de Spondias tuberosa oriundas de frutos cosechados en cuatro estadios de maduración y almacenadas 
almacenamiento, principalmente bajo condiciones ambiente; pero, para altura de plantas (Figura 4D), hubo efecto lineal para ambientes hasta el último periodo del almacenamiento ( 8 meses), siendo este tiempo considerado corto para un posible buen desarrollo de las plantas. Por otro lado, para el diámetro (Figura 4E) y longitud de túbera (Figura 4F), hubo efectos semejantes al almacenamiento en la calidad de las semillas de Spondias sp (Figure 3E e F).

Esa reducción de valores a partir de los seis meses de almacenamiento se debe probablemente, a factores físicos, tales como, impedimento del crecimiento longitudinal de la raíz, una vez que la profundidad del sustrato fue de apenas $10 \mathrm{~cm}$ de alto, siendo éste un factor limitante al desarrollo normal de la túbera. Observase aún que, mientras el crecimiento longitudinal de la túbera fue limitado (Figura 4F), el diámetro de las mismas sigue creciendo con el tiempo de almacenamiento (Figura 4E), lo que se hace cree en una posible necesidad de aumento en el tiempo de evaluación de semillas de Spondias tuberosa, almacenadas, como por ejemplo, haciéndose uso de un mayor espacio de tiempo y de mayor profundidad de sustrato.

\section{CONCLUSIONES}

1. El último periodo de almacenamiento (ocho meses), fue lo más adecuado a una mejor uniformidad de germinación de las semillas:

2. El ambiente natural es el ideal para almacenamiento de semillas de Spondias tuberosa desde la cosecha de los frutos hasta su plantío:

3. Los estadios de maduración de frutos maduros y muy maduros, fueron los más adecuados para optimizar una buena germinación inicial de las semillas:

4. El mayor vigor fue alcanzado por semillas oriundas de frutos maduros.

\section{LITERATURA CITADA}

Alvarenga, E.M.; Silva, R.F.; Araújo, E.F. Influência da idade, armazenamento pós-colheita dos frutos na qualidade fisiológica. Horticultura Brasileira, Brasília, v.2, n.2, p.5-8, 1984.

Araújo, F.P.; Santos, C.A.F.; Cavalcanti, N.B.; Rezende, G.M. Influência do período de armazenamento das sementes de umbuzeiro na sua germinação e no desenvolvimento de plântula. Revista Brasileira de Armazenamento, Viçosa, v.1, n.26, p.36-39, 2001.

Campos, C.O. Estudo da quebra de dormência da semente de umbuzeiro (Spondias tuberosa Arruda Câmara). Fortaleza: UFC, 1996. 71p. Dissertação Mestrado

Cavalcanti, N.B.; Resende, G.M.; Brito, L.T.L.; Lima, J.B. Extrativismo do imbuzeiro (Spondias tuberosa Arruda Câmara) como fonte alternativa de renda para pequenos produtores no semi-árido nordestino: um estudo de caso. Ciência e Agrotécnica, Lavras, v.20, n.4, p.525-528, 1996.
Cavalcanti, N.B.; Resende, G.M.; Brito, L.T.L. Desenvolvimento do imbuzeiro (Spondias tuberosa Arruda Câmara) na região semi-árida do nordeste brasileiro. Ciência e Agrotécnica, Lavras, v.23, n.1, p.212-213, 1999.

Cavalcanti, N.B.; Resende, G.M.; Brito, L.L.T. Período de ocorrência da frutificação do umbuzeiro na região semi-árida de Pernambuco. In: Congresso Brasileiro de Fruticultura, 8 , 2004. Florianópolis. Anais... Florianópolis: 2004. CD Rom

Costa, N.P. da. Caracterização de frutos e germinação de endocarpos de umbu (Spondias tuberosa Arruda Câmara). Areia: UFPB, 1999. 65p. Dissertação Mestrado

Gondim, T.M.S.; Silva, H.; Silva, A.Q. Período de ocorrência da formação de xilopódio em plantas de umbu (Spondias tuberosa Arruda Câmara) propagadas sexuada e assexuadamente. Revista Brasileira de Fruticultura, Jaboticabal, v.13, n.2, p.35-40, 1991.

Lederman, I.E.; Gonzaga Neto, L.; Bezerra, J.E.F. Indução de germinação de sementes de umbu (Spondias tuberosa Arruda Câmara) através de tratamentos físicos, químicos e mecânicos. Revista Brasileira de Fruticultura, Jaboticabal, v.11, n.3, p.27-32, 1989.

Lozano, N.B. Desarrollo y anatomia de fruto del jabo (Spondias mombin L.). Caldasia, Bogotá, v.14, n.68-70, p.465-490, 1986.

Martins, L.P.; Silva, S.M.; Alves, R.E.; Filgueiras, H.A.C. Desenvolvimento de frutos de ciriguela (Spondias purpurea L.). Revista Brasileira de Fruticultura, Jaboticabal, v.25, n.1, p.11-14, 2003a.

Martins, L.P.; Silva, S.M.; Alves, R.E.; Filgueiras, H.A.C. Fisiologia do dano pelo frio em ciriguela (Spondias purpurea L.). Revista Brasileira de Fruticultura, Jaboticabal, v.25, n.1, p.23 -26, 2003 b.

Mendes, B.V. Umbuzeiro (Spondias tuberosa Arruda Câmara): importante frutífera do semi-árido. Mossoró: ESAM, 1990. 67p. Coleção Mossoroense - Série C, v.564

Silva, A.Q.; Moura, F.T.; Cavalcanti, C.L. Florescimento e frutificação do umbuzeiro (Spondias tuberosa Arruda Câmara) no Cariri paraibano. In: Reunião Nordestina de Botânica, 12, João Pessoa, 1998. Anais... João Pessoa - UFPB, 1998.57p.

Silva, A.Q.; Silva, M.A.G.O. Observações morfológicas e fisiológicas sobre Spondias tuberosa Arr. Câm. In: Congresso Nacional de Botânica, 25, 1974. Mossoró. Anais... Mossoró: ESAM, 1974, p.5-15.

Souza, F.X. Spondias Agro-industriais e os seus métodos de propagação. Fortaleza: EMBRAPA - CNPAT, 1999.

Souza, R.P.; Filgueiras, H.A.C.; Costa, J.T.A.; Alves, R.E.; Oliveira, A.C. Armazenamento da ciriguela (Spondias purpurea L.) sob atmosfera modificada e refrigeração. Revista Brasileira de Fruticultura, Jaboticabal, v.22, n.3, p.334$338,2000$. 